\title{
ERLIN1 mutations cause teenage-onset slowly progressive ALS in a large Turkish pedigree
}

\author{
Ceren Tunca $\mathbb{D}^{1} \cdot$ Fulya Akçimen $^{1} \cdot$ Cemre Coşkun ${ }^{1} \cdot$ Aslı Gündoğdu-Eken ${ }^{1} \cdot$ Cemile Kocoglu $^{1}$ Betül Çevik ${ }^{2}$. \\ Can Ebru Bekircan-Kurt ${ }^{3} \cdot{\text { Ersin } \operatorname{Tan}^{3} \cdot \text { A. Nazlı Başak }}^{1}$
}

Received: 5 October 2017 / Revised: 26 December 2017 / Accepted: 5 January 2018 / Published online: 16 February 2018

(c) European Society of Human Genetics 2018

\begin{abstract}
Amyotrophic lateral sclerosis (ALS) is a late-onset motor neuron disease with mostly dominant inheritance and a life expectancy of 2-5 years; however, a quite common occurrence of atypical forms of the disease, due to recessive inheritance, has become evident with the use of NGS technologies. In this paper, we describe a family with close consanguinity for at least four generations, suffering from a slowly progressive form of ALS. Spastic walking is observed since teenage years, while bulbar symptoms start much later, at the fifth or sixth decade of life. Patients usually die because of respiratory failure. Using whole-exome sequencing, we identified a novel homozygous p.(Val94Ala) (c.281T>C) (NG_052910.1) (NM_006459) variation in the endoplasmic reticulum lipid raft associated protein 1 (ERLINI) gene, which segregates with the disease in the family. Here we suggest that ERLIN1 variants, previously shown in juvenile hereditary spastic paraplegia cases, may also be the cause of a slowly progressive early-onset ALS, starting with upper motor neuron features and developing into classical ALS with the addition of lower motor neuron dysfunction. We also demonstrate that ATPbinding cassette subfamily C member 2 (ABCC2) gene, responsible for hyperbilirubinemia, is linked to ERLINI.
\end{abstract}

\section{Introduction}

Motor neuron disorders are complex diseases with diverse overlapping phenotypes ranging from infantile to adultonset or from mild to lethal forms, making clinical diagnosis challenging. The heterogeneity of amyotrophic lateral sclerosis (ALS) within itself is also highlighted by the growing list of genes linked to both familial and sporadic forms of the disease [1]. The era of high-throughput

Electronic supplementary material The online version of this article (https://doi.org/10.1038/s41431-018-0107-5) contains supplementary material, which is available to authorized users.

$\triangle$ A. Nazlı Başak

basak@boun.edu.tr

1 Department of Molecular Biology and Genetics, Suna and İnan Kıraç Foundation, Neurodegeneration Research Laboratory (NDAL), Boğaziçi University, Istanbul, Turkey

2 Department of Neurology, Gaziosmanpaşa University Medical School, Tokat, Turkey

3 Department of Neurology, Hacettepe University Medical School, Ankara, Turkey sequencing enabled more detailed and sophisticated research in understanding the genetic make-up behind these diseases, especially in cases where the hereditary component is strengthened by consanguinity [2-4]. The increased pleiotropy observed among motor neuron disease genes and the overlapping phenotypes point to a thin line between different phenotypes, which is often crossed during the course of the disease [5-7]. Here, we report the results of exome sequencing analyses in a large consanguineous Turkish pedigree with five living affected members displaying slowly progressive teenage-onset motor neuron dysfunction starting with walking difficulties, and gradually developing into a severe form of ALS with lower motor neuron involvement during mid-life.

\section{Subjects and methods}

The female index patient (V.12), at the age of 25, was referred to our center with young-onset ALS and a family history of ALS in the deceased father (IV.24) as well as in the paternal aunt (IV.22) and grandfather (III.18) (Fig. 1). The grandfather was reported to suffer from walking difficulties starting around the age of 35; he then developed 


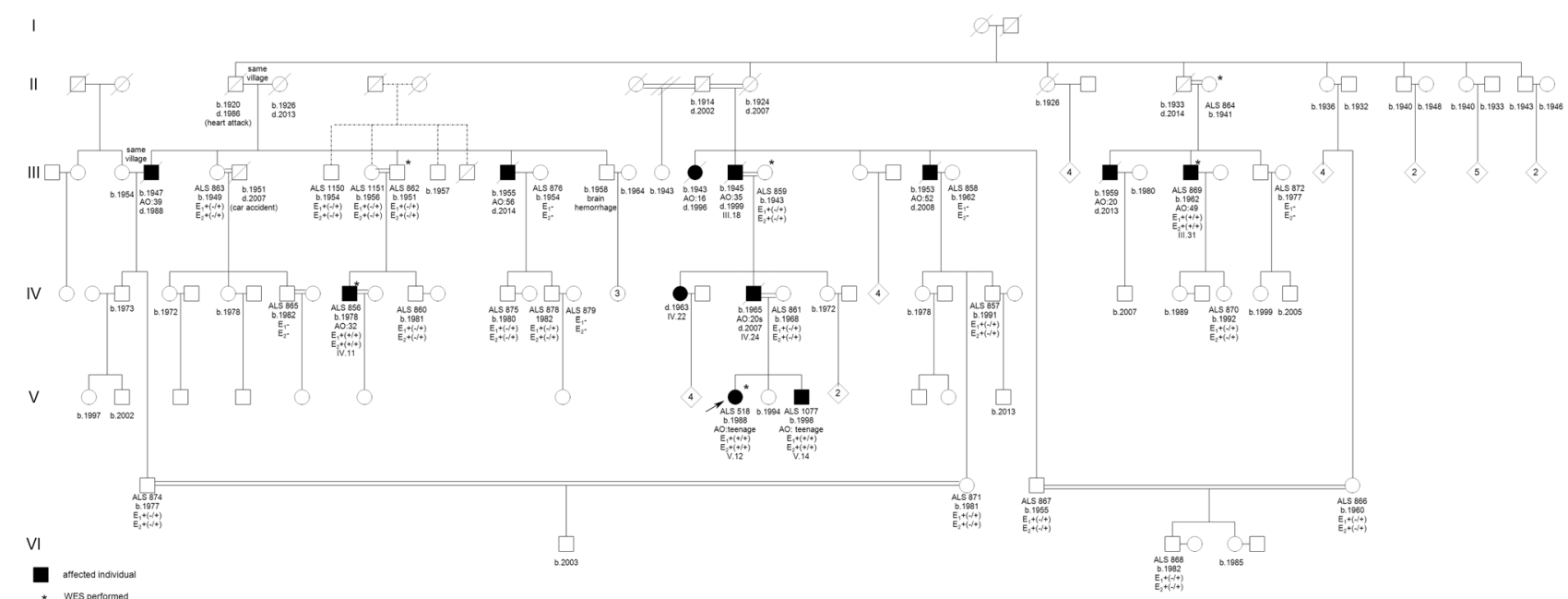

Fig. 1 Pedigree of the family with ERLIN1 variation. The double lines between spouses indicate consanguinity. Segregation of the identified variations $\mathrm{E}_{1}$ (ERLIN1 c.281T >C, p.(Val94Ala), NM_006459) and $\mathrm{E}_{2}$ (ABCC2 c.2714G > T, p.(Arg905Ile), NM_000392) are designated. The individual IDs in the pedigree (e.g., ALS 518) are laboratory

swallowing problems, which led to his death at 54 . The father limped mildly after returning from military service at the age of 20 . He underwent surgery with spinal stenosis diagnosis; however, his symptoms worsened. The electroneuromyography showed fibrillation and fasciculation in genioglossus, right first dorsal interossei, biceps, quadriceps, gastrocnemius, and tibialis anterior muscles. Upon these findings, he was diagnosed with ALS and succumbed to the disease at the age of 42 . His daughter, our index case, had complaints of abnormal gait during her late teens and was referred to the expert neurologist (E.T.), who had also diagnosed and followed her deceased father. At the time of her genetic diagnosis, the neurological examination depicted increased deep tendon reflexes in both lower extremities and atrophy in left interosseous muscles, electrophysiological evaluation revealed asymmetrical upper and lower motor neuron signs with fibrillation and fasciculation in genioglossus, right first interossei, biceps, and both tibialis anterior muscles, confirming definite ALS according to El Escorial criteria. On the other hand, her younger, 15year-old brother (V.14) only had slowed motor evoked potential in asymmetrically bilateral lower limbs.

Considering the apparently vertical inheritance pattern in this family, common ALS genes (C9ORF72, SOD1, $T A R D B P, F U S$, and $U B Q L N 2$ ) were ruled out prior to whole-exome sequencing. However, upon detailed family investigation, the recessive inheritance due to high consanguinity in every generation became evident (Fig. 1). Two additional male individuals in the pedigree also had mild gait abnormalities (III.31 and IV.11). Neurological examination of III.31, 52-year-old, showed hyperreflexia in internal IDs, do not designate any phenotype, and are for the purpose of finding individuals in the LOVD webpage. * shows the individuals subjected to whole-exome sequencing analysis. AO age of onset, b date of birth, $d$ date of death

the legs, positive Babinski sign on the right, and unresponsive plantar reflex on the left foot with bilateral clonus. Patient IV.11 has difficulty in step climbing.

A detailed explanation of the methods is available in the Supplementary Information. The phenotype and variant data can be found online (https://databases.lovd.nl/shared/ genes/ERLIN1) (patient IDs 00143683, 00143789, 00143791, and 00143792).

\section{Results and discussion}

Whole-exome sequencing was performed in three affected and three unaffected members (indicated by an asterisk* in the pedigree) of a large and highly inbred family from the Black Sea region of Turkey (Fig. 1). After filtration for autosomal recessive inheritance pattern 21 gene variants remained, only two of which (in ERLIN1 and ABCC2) had a MAF lower than 0.01 (Supplementary Table 1). The novel homozygous p.(Val94Ala) (c.281T >C) (NM_006459) variation in the endoplasmic reticulum lipid raft associated protein 1 (ERLINI) gene, co-segregating with the disease in the family across a genetic distance of at least nine meioses to attain homozygous state, was suggested to be the more probable candidate for the phenotype in question. The homozygous presence of the ERLINI variation was validated by Sanger sequencing in four affected family members (III.31, IV.11, V.12, and V.14). Additional 22 family members were shown to be either heterozygous or wild-type for the variation, are symptom-free at present and not expected to develop the disease in view of their genotype. Homozygosity 
Fig. 2 The conservation of the valine residue at position 94 (in bold) of ERLIN1 protein among 10 species

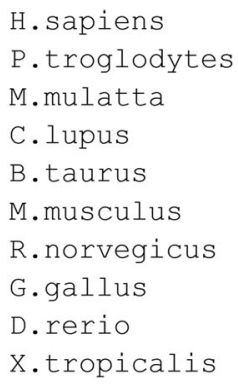

51 YHIMLPFITTFRSVQTLQTDEVKNVPCGTSGGVMIYIDRIEVVNMLAPY 100 51 YHIMLPFITTFRSVQTTLQTDEVKNVPCGTSGGVMIYIDRIEVVNMLAPY 100 51 YHIMLPEITTFRSVOTTLOTDEVKNVPCGTSGGVMIYIDRIEVVNMLAPY 100 51 YHIMLPFITTFRSVQTTLQTDEVKNVPCGTSGGVMIYIDRIEVVNMLAPC 100 51 YHIMLPEITTERSVQTTLQTDEVKNVPCGTSGGVMIYIDRIEVVNMLAPC 100 51 YHIMLPFITTERSVQTTLQTDEVKNVPCGTSGGVMIYIDRIEVVNMLAPY 100 51 YHIMLPFITTFRSVQTTLQTDEVKNVPCGTSGGVMIYIDRIEVVNMLAPY 100 51 YHIMLPFITTFKSVOTTLQTDEVKNVPCGTSGGVMIYIDRIEVVNKLAPY 100 49 YHIMLPFITSYRSVQTTLQTDEIKNVPCGTSGGVMIYFDRIEVVNMLIPT 98 49 YHIMFPFITYERVQTTLQTDEVKNVPCGTSGGVMIYEDRIEVVNMLTPS 98 mapping, performed from the exome data, detected the ERLIN1 locus in all affected individuals, but not in the controls (Supplementary Table 2). The valine to alanine substitution at position 94 of ERLIN1, located in a highly conserved region, was predicted to be disease-causing, deleterious, and possibly damaging by MutationTaster, SIFT, and PolyPhen2 (Fig. 2). No other gene associated with autosomal recessive hereditary spastic paraplegia (ARHSP) or ALS was mutated in the affected individuals.

The other homozygous change remaining after filtration for mode of inheritance and minor allele frequency lower than 0.01 was the p.(Arg905Ile) $\quad(\mathrm{c} .2714 \mathrm{G}>\mathrm{T})$ (NG_011798.1) (NM_000392) variation in the ATPbinding cassette subfamily $C$ member 2 (ABCC2) gene reported to be associated with Dubin-Johnson syndrome and characterized by hyperbilirubinemia (MAF:0.000049) [8]. The $A B C C 2$ gene resides in the same homozygous region with ERLIN1. Segregation analysis for this variation showed that the allelic states (genotypes) for ERLIN1 and $A B C C 2$ genes were the same for all tested family members (Fig. 1). Pair-wise linkage disequilibrium for ERLIN1 and $A B C C 2$ variations was estimated by the squared genotype correlation coefficient $\left(r^{2}\right)$ in our in-house exome database of 420 individuals. An estimate of $r^{2}=1$ indicates that the two variations are in complete linkage disequilibrium. Also, according to the HapMap data, ABCC2 and ERLIN1 reside in $0.2 \mathrm{cM}$ distance, mapped to $127.3 \mathrm{cM}$ and $127.5 \mathrm{cM}$ of chromosome 10, respectively. To the best of our knowledge, $A B C C 2$ does not seem to be responsible for the motor neuron phenotype seen in our family, although it segregates with the disease gene. However, more evidence is needed to understand the effect of the $A B C C 2$ variant, as well as many other modifier variants on the development, manifestation, and progression of complex neurological phenotypes.

ERLIN1 encodes for a prohibitin-domain-containing protein located in the ER membrane that forms a ringshaped complex with ERLIN2. ERLIN1/2 complex is implicated in endoplasmic reticulum-associated degradation (ERAD) control and is responsible for forming a bridge between the substrates recognized by the ER lumen and the E3 ligases located in the ER membrane [9]. ER stress is one of the major pathogenic events in ALS, as protein products of ALS-causing genes like optineurin, ubiquilin-2, valosincontaining protein, and TANK-binding kinase-1 are involved in several steps of the ERAD pathway, which includes the recognition of misfolded proteins, ubiquitination, and transportation of the cargo, and degradation through proteasome or autophagosome [10, 11].

Three different ERLIN1 mutations have been previously identified in seven patients with pure ARHSP from three independent families all having infantile-onset symptoms, except one patient with an age of onset of 13 [12]. Two of these variations, are located in the low complexity domain of the protein, whereas one is in the prohibitin domain like our variation.

Mutations in the ERLIN1 gene, associated with spastic paraplegia 62 (SPG62), were so far thought to cause a pure form of HSP that primarily affects the upper motor neurons [13]. As a novel observation, the ERLIN1 gene variant in our index case and her father have led to definitive ALS according to El Escorial criteria. In the family presented, the ages of onset and short survival times due to lower motor neuron involvement later in the disease resemble typical adult-onset ALS in all patients investigated.

Some forms of HSP are clinically hard to distinguish from ALS. SPG11-based disease is the best example, where clinical diagnosis may differ even among sibs as either ARHSP or autosomal recessive juvenile ALS [14, 15]. There are key clinical features that are present in one case, but not in others, which draw a line between two clinical diagnoses, but may change even during the course of the disease. We therefore strongly suggest that the ERLIN1 variation in our family results in a clinical presentation, which starts as a mild form of HSP and progresses to ALS.

ALS is a late-onset disease and juvenile patients are rare. However, in Turkey, due to high first-cousin consanguinity, young $(<20$ year-old) cases with recessive mutations are common [16]. Interestingly and commonly, these juvenile cases with consanguinity in the family present with additional novel clinical features that are not common in classical ALS [17]. Making a firm diagnosis in such cases is difficult for clinicians, since overlapping symptoms blur the boundaries between related diseases [18]. This study shows that NGS (Next Generation Sequencing) allows 
complicated cases to be investigated in an unbiased manner and futher extends the clinical spectrum of ERLIN1-based disease from ARHSP to autosomal recessive juvenile ALS. The steady increase in the number of novel genetic players identified in hereditary neurologic diseases will improve our understanding of the mechanisms underlying these diseases for future therapeutic approaches.

Acknowledgements We thank Dr. Şule Ekim, Ufuk Çopur, and all other family members for hosting us in their village and for their cooperation and Irmak Şahbaz from NDAL for her excellent technical assistance. Suna and İnan Kıraç Foundation is acknowledged for the generous funding of the study. This study was accomplished at Boğaziçi University.

\section{Compliance with ethical standards}

Conflict of interest The authors declare that they have no conflict of interest.

\section{References}

1. Ghasemi M, Brown RH. Genetics of amyotrophic lateral sclerosis. Cold Spring Harb Perspect Med. 2017;7:1-38.

2. Foo JN, Liu JJ, Tan EK. Whole-genome and whole-exome sequencing in neurological diseases. Nat Rev Neurol. 2012;8:508-17.

3. Koboldt DC, Steinberg KM, Larson DE, Wilson RK, Mardis ER. The next-generation sequencing revolution and its impact on genomics. Cell. 2013;155:27-38.

4. Bras J, Guerreiro R, Hardy J. Use of next-generation sequencing and other whole-genome strategies to dissect neurological disease. Nat Rev Neurosci. 2012;13:453-64.

5. Guerreiro R, Bras J, Hardy J, Singleton A. Next generation sequencing techniques in neurological diseases: redefining clinical and molecular associations. Hum Mol Genet. 2014;23:47-53.
6. Synofzik M, Smets K, Mallaret M, et al. SYNE1 ataxia is a common recessive ataxia with major non-cerebellar features: a large scale multi-centre study. Brain. 2016;139:1378-93.

7. Frasquet M, Va JF, Sevilla T. The role of DNAJB2 in amyotrophic lateral sclerosis. Brain. 2017;139:1-2.

8. Kajihara S, Hisatomi A, Mizuta T, Hara T, Ozaki I, Wada I, et al. A splice mutation in the human canalicular multispecific organic anion transporter gene causes Dubin-Johnson syndrome. Biochem Biophys Res Commun. 1998;457:454-7.

9. Olzmann JA, Kopito RR, Christianson JC. The mammalian endoplasmic reticulum-associated degradation system. Cold Spring Harb Perspect Biol. 2013;5:a013185.

10. Taylor JP, Brown RH, Cleveland DW. Decoding ALS: from genes to mechanism. Nature. 2016;539:197-206.

11. Cirulli ET, Lasseigne BN, Petrovski S, et al. Exome sequencing in amyotrophic lateral sclerosis identifies risk genes and pathways. Science. 2015;347:1436.

12. Novarino G, Fenstermaker AG, Zaki MS, et al. Exome sequencing links corticospinal motor neuron disease to common neurodegenerative disorders. Science. 2014;343:506-11.

13. Parodi L, Fenu S, Stevanin G, Durr A. Hereditary spastic paraplegia: more than an upper motor neuron disease. Rev Neurol. 2017;173:352-60.

14. Daoud H, Zhou S, Noreau A, et al. Exome sequencing reveals SPG11 mutations causing juvenile ALS. Neurobiol Aging. 2012;33:5-9.

15. Iskender C, Kartal E, Akcimen F, et al. Turkish families with juvenile motor neuron disease broaden the phenotypic spectrum of SPG11. Neurol Genet. 2015;1:e25.

16. Özoğuz A, Uyan Ö, Birdal G, et al. The distinct genetic pattern of ALS in Turkey and novel mutations. Neurobiol Aging. 2015;36:1764.e9-18.

17. Ben Hamida M, Hentati F, Ben Hamida C. Hereditary motor system diseases (chronic juvenile amyotrophic lateral sclerosis). Brain. 1990;113:347-63.

18. Deschauer M, Gaul C, Behrmann C, Prokisch H, Zierz S, Haack TB. C19orf12 mutations in neurodegeneration with brain iron accumulation mimicking juvenile amyotrophic lateral sclerosis. J Neurol. 2012;259:2434-9. 